\title{
BROOKHTWEN
}

NATIONAL LABORATORY

BNL-114315-2017-IR

\section{Brief History of Iron Evaluations in the ENDF/B Library}

\author{
D.A. Brown
}

September 06, 2017

\author{
National Nuclear Data Center \\ Brookhaven National Laboratory
}

\author{
U.S. Department of Energy \\ USDOE Office of Science (SC), \\ Nuclear Physics (NP) (SC-26)
}

Notice: This manuscript has been authored by employees of Brookhaven Science Associates, LLC under Contract No.DE-SC0012704 with the U.S. Department of Energy. The publisher by accepting the manuscript for publication acknowledges that the United States Government retains a non-exclusive, paid-up, irrevocable, world-wide license to publish or reproduce the published form of this manuscript, or allow others to do so, for United States Government purposes. 


\section{DISCLAIMER}

This report was prepared as an account of work sponsored by an agency of the United States Government. Neither the United States Government nor any agency thereof, nor any of their employees, nor any of their contractors, subcontractors, or their employees, makes any warranty, express or implied, or assumes any legal liability or responsibility for the accuracy, completeness, or any third party's use or the results of such use of any information, apparatus, product, or process disclosed, or represents that its use would not infringe privately owned rights. Reference herein to any specific commercial product, process, or service by trade name, trademark, manufacturer, or otherwise, does not necessarily constitute or imply its endorsement, recommendation, or favoring by the United States Government or any agency thereof or its contractors or subcontractors. The views and opinions of authors expressed herein do not necessarily state or reflect those of the United States Government or any agency thereof. 


\title{
Brief History of Iron Evaluations in the ENDF/B Library
}

\author{
D.A. Brown,${ }^{1}$ M. Herman,${ }^{1}$ and G.P.A. Nobre ${ }^{1}$ \\ ${ }^{1}$ National Nuclear Data Center, Brookhaven National Laboratory, Upton, NY 11973, USA*
}

(Dated: September 14, 2017)

The report summarizes the development of the iron evaluations from ENDF/B-V to present.

\section{CONTENTS}

I. Introduction

II. $\mathrm{ENDF} / \mathrm{B}-\mathrm{V}$
A. ENDF/B-V.0 (1979)
B. ENDF/B-V.1 (1983)
C. ENDF/B-V.2 (1985)

III. ENDF/B-VI
A. ENDF/B-VI.0 (1990)
B. ENDF/B-VI.1 (1991)
C. ENDF/B-VI.2 (1993) -VI.3 (1995) -VI.4 (1996)
D. ENDF/B-VI.5 (1997-1998)
E. ENDF/B-VI.6 (1998-1999)
F. ENDF/B-VI.7 (1999-2000)
G. ENDF/B-VI.8 (2001)

IV. ENDF/B-VII
A. ENDF/B-VII.0 (2006)
B. ENDF/B-VII.1 (2011)

\section{ENDF/B-VIII}
A. ENDF/B-VIII.0 $\beta 0$ (8 Apr. 2016)
B. ENDF/B-VIII.0 $\beta 1$ (25 Apr. 2016)
C. ENDF/B-VIII.0 22 (19 Aug. 2016)
D. ENDF/B-VIII.0 $\beta 3$ (2 Nov. 2016)
E. ENDF/B-VIII.0ß33.1 (19 Jan. 2017)
F. ENDF/B-VIII.0 $\beta 4$ (28 Feb. 2017)

1

1

1

6

6

6

6

7

7

7

8

8

8

8

VI. Acknowledgments

References

\section{INTRODUCTION}

This report outlines the history of ENDF/B evaluations of the iron isotopes. Our detailed history extends

\footnotetext{
* Corresponding author: dbrown@bnl.gov
}

back to ENDF/B-V, when isotopic evaluations were first introduced in the ENDF/B library. This history ends at the ENDF/B-VIII.0 $\beta 4$ evaluation as the ENDF/BVIII.0 evaluations are described in [1] and the ENDF/BVIII.0 34.1 evaluations are basically the released files.

Before ENDF/B-V, the ENDF/B library only contained a natural iron evaluation. Details of the ENDF/BIII (1972) natural iron evaluation are described in ORNL4617 (ENDF-139) [2] and details of both the ENDF/B-IV (1975) and ENDF/B-V (1979) evaluations are described in ORNL/TM-7523 (ENDF-302) [3].

Figures 1-4 provide a graphical overview of the history of each ENDF evaluation.

\section{ENDF/B-V}

In ENDF/B-V, the resonances in this file were split into the isotopic files while new new fast regions evaluations for all the isotopes were provided. After this release, only isotopic evaluations are present in ENDF.

\section{A. ENDF/B-V.0 (1979)}

${ }^{n a t} \mathbf{F e}$ [Fu (ORNL)]The ENDF/B-V.0 natural iron evaluation contained several notable features:

- The resonance parameters for ${ }^{54} \mathrm{Fe},{ }^{56} \mathrm{Fe}$ and ${ }^{57} \mathrm{Fe}$ from $1 \mathrm{keV}$ to $400 \mathrm{keV}$ were examined in detail by C.M.Perey [8]. Parameters for ${ }^{58} \mathrm{Fe}$ were taken from the ENDF/B-V dosimetry file [9].

- In the total and capture cross sections, broad structures in the resonance range $(155 \mathrm{eV}$ to $400 \mathrm{keV})$ were kept unchanged from ENDF/B-IV values. Narrow resonances were better defined.

- The capture cross sections from $155 \mathrm{eV}$ to $400 \mathrm{keV}$ are well-represented by the resonance parameters. From $400 \mathrm{keV}$ to $1 \mathrm{MeV}$ average cross sections measured by Allen et al. [10, 11], which agrees with those of Le Rigoleur [12], were adopted. Above 2 $\mathrm{MeV}$, the capture cross section was increased. 


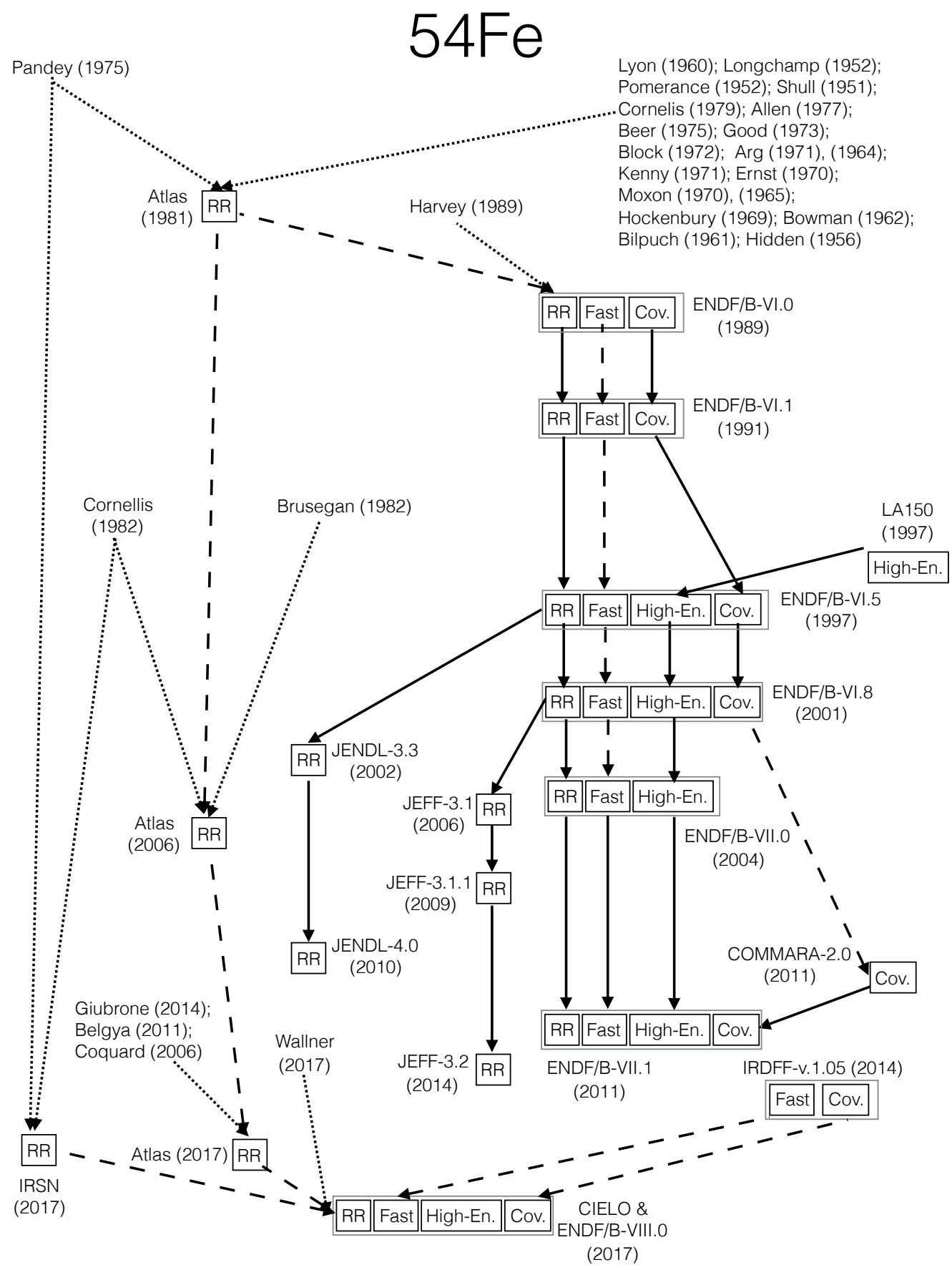

FIG. 1. Provenance of the CIELO and ENDF/B-VIII ${ }^{54}$ Fe evaluation. Each major component of the evaluation from all libraries that fed the current evaluation are shown. Experiments that were included in the resonance evaluation are listed explicitly even if they are not mentioned in the main text. These data are referenced in the Atlas of Neutron Resonances [4]. We note that various revisions of the Atlas of Neutron Resonances [4-6] played key roles in the development of several data libraries. In this figure, the dotted line represents the addition of experimental data into the evaluation, the dashed line represents a modification to an existing evaluation and a solid line represents the adoption of an evaluation, unchanged. 


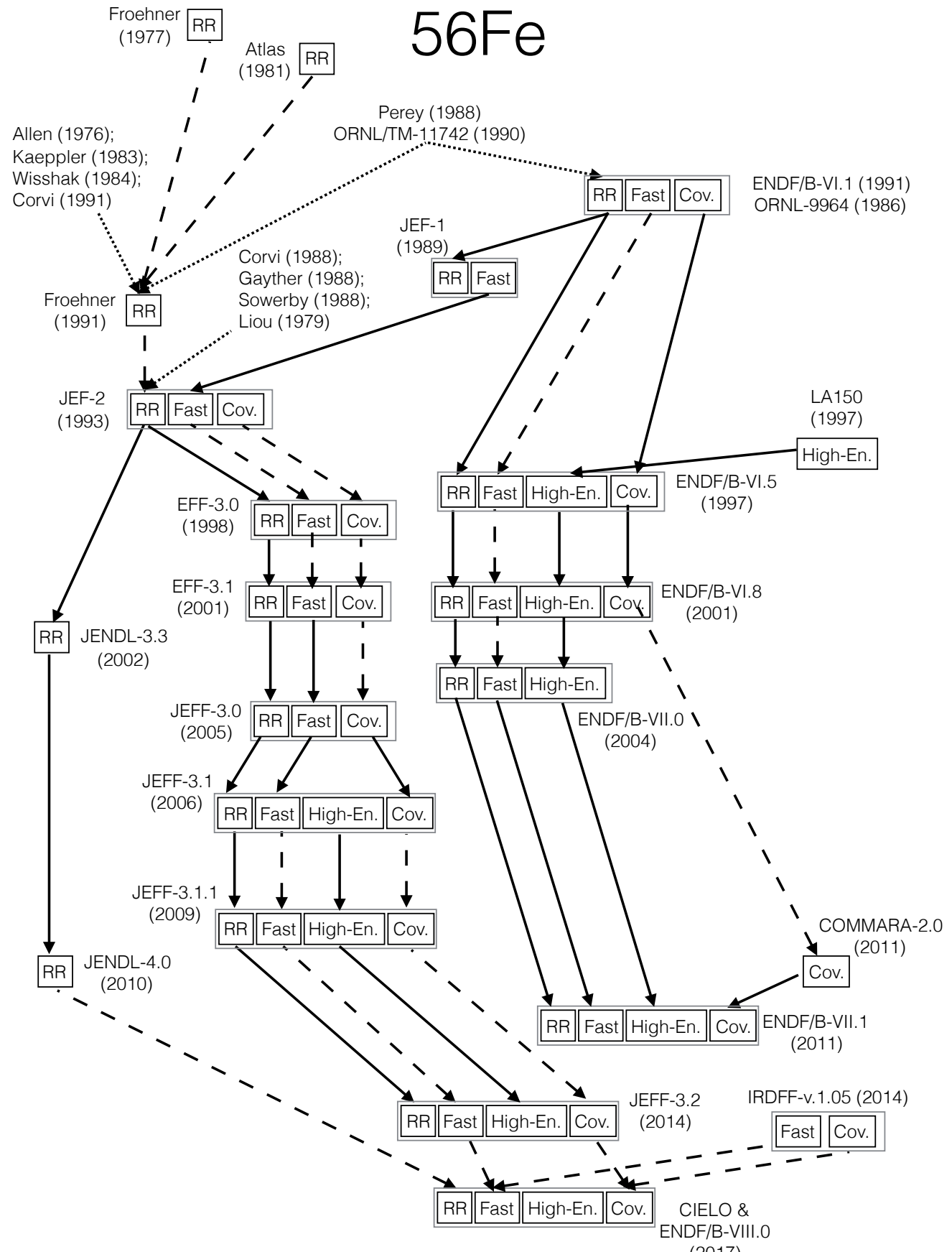

(2017)

FIG. 2. Provenance of the CIELO and ENDF/B-VIII ${ }^{56}$ Fe evaluation. Each major component of the evaluation from all libraries that fed the current evaluation are shown. Experiments that were included in the resonance evaluation are listed explicitly even if they are not mentioned in the main text. These data are referenced in the Atlas of Neutron Resonances [4]. The new CIELO resonance evaluation is based in the JENDL-4.0 resonance evaluation. The fast region of the CIELO evaluation is based on a combination of new EMPIRE calculation and IRDFF and JEFF evaluations. In this figure, the dotted line represents the addition of experimental data into the evaluation, the dashed line represents a modification to an existing evaluation and a solid line represents the adoption of an evaluation, unchanged. 


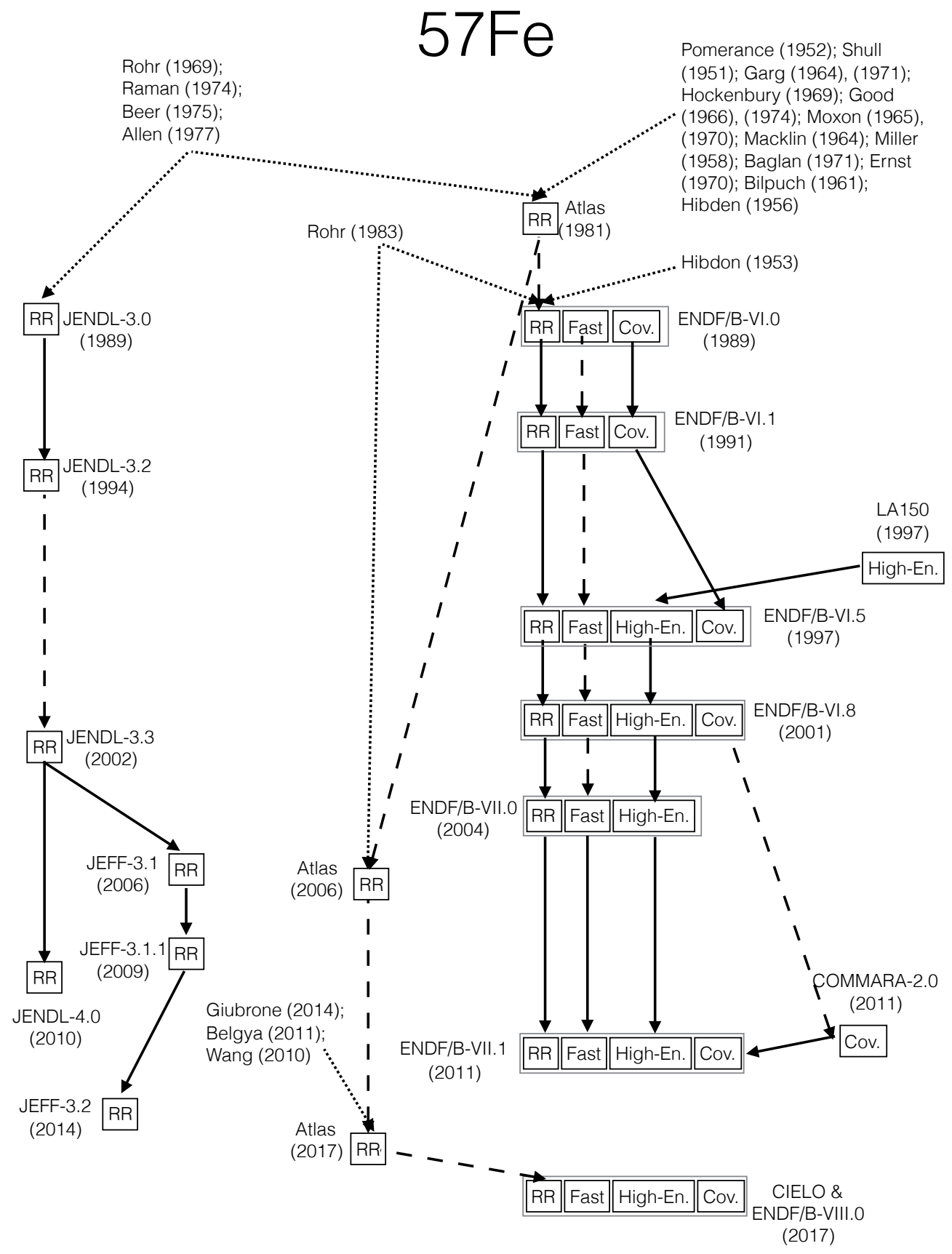

FIG. 3. Provenance of the CIELO and ENDF/B-VIII ${ }^{57}$ Fe evaluation. Each major component of the evaluation from all libraries that fed the current evaluation are shown. Experiments that were included in the resonance evaluation are listed explicitly even if they are not mentioned in the main text. These data are referenced in the Atlas of Neutron Resonances [4]. The new evaluation is a reformatted (into $\mathrm{LRF}=7$ ) and tweaked version of what is available in the new Atlas [4]. In this figure, the dotted line represents the addition of experimental data into the evaluation, the dashed line represents a modification to an existing evaluation and a solid line represents the adoption of an evaluation, unchanged. 


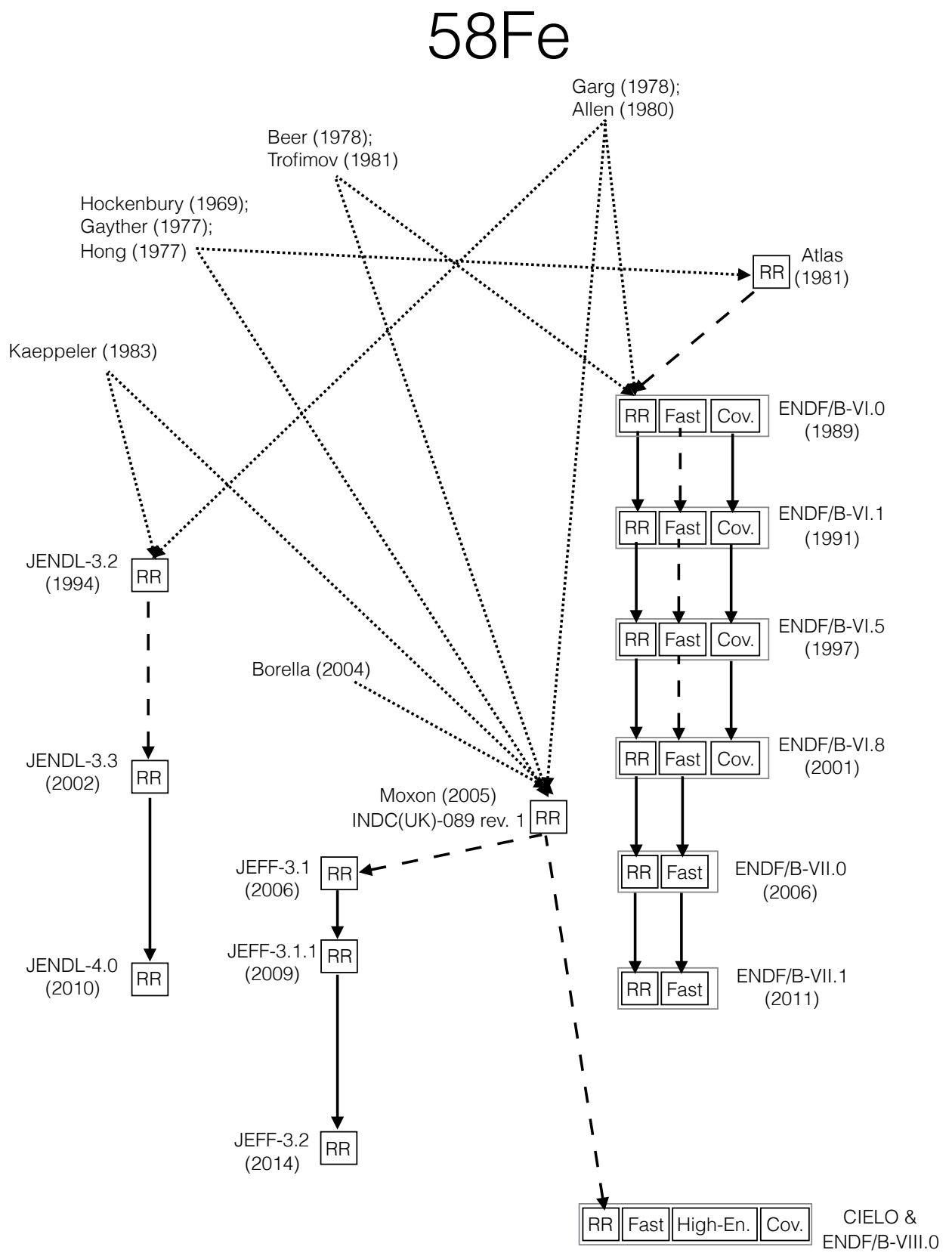

(2017)

FIG. 4. Provenance of the CIELO and ENDF/B-VIII ${ }^{58}$ Fe evaluation. Each major component of the evaluation from all libraries that fed the current evaluation are shown. Experiments that were included in the resonance evaluation are listed explicitly even if they are not mentioned in the main text. These data are referenced in the Atlas of Neutron Resonances [4] and in Moxon's writeup [7]. The new evaluation's resonances are taken from Moxon [7]. In this figure, the dotted line represents the addition of experimental data into the evaluation, the dashed line represents a modification to an existing evaluation and a solid line represents the adoption of an evaluation, unchanged. 
- The inelastic cross section corresponding to the first excited state of ${ }^{56} \mathrm{Fe}$ was revised according to the recommendation of Kinney and Perey [13] up to $1300 \mathrm{keV}$.

- The $(n, \gamma)$ cross section was substantially increased to go through a data point near $14 \mathrm{MeV}$ [14].

- For the elastic angular distribution, high-resolution data of Kinney and Perey [15] from $40 \mathrm{keV}$ to 1.4 MeV were adopted. The broad trend of the Legendre coefficients are in good agreement with $\mathrm{ENDF} / \mathrm{B}-\mathrm{IV}$ values.

- All gammas were lumped in the total inelastic cross section $(\mathrm{MT}=3)$ and were taken from Chapman and Morgan [16]. This is a repeat measurement using ORELA which resolves a long-standing discrepancy. The lowest three neutron energies (up to $2.122 \mathrm{MeV}$ ) are for capture gamma rays.

- Covariances for the resonance parameters evaluated by C.M.Perey [8].

${ }^{56} \mathbf{F e}$ [Fu (ORNL)] Experimental data available since the evaluation of Fe-56 (n,p) cross sections by Dudey and Kennerley [17] were reviewed. Smith and Meadows [18] reported new data from 4 to $10 \mathrm{MeV}$, an energy range most important for application of this reaction to fission reactor dosimetry. Vonach [19] has recently called for attention to his data [20] from 13 to $15 \mathrm{MeV}$. Further checks [19] resulted in a reduction of the data errors from $4 \%$ to $2.5 \%$. The data had not been used in the Dudey and Kennerley evaluation but are 2 to $3 \%$ lower than the evaluated values. Three single-energy measurements [21-23] near 14.6 MeV have been reported. The one by Dyer [21] is $1 \%$ higher than the ENDF/B-IV value, Robertson $7 \%$ higher, and Qaim 4\% lower. Inclusion of the new data in a subjective analysis results in up to $10 \%$ reduction from the ENDF/B-IV values from threshold to $6 \mathrm{MeV}, 2-3 \%$ reduction form 6 to $10 \mathrm{MeV}$, and $1-2 \%$ reduction from 13-15 MeV. The data of Smith and Meadows are relative to ENDF/B-IV U-238(n,f) cross sections and had been renormalized to ENDF/B-V values before being used in the present evaluation. The effect on the calculated fission spectrum average is estimated to be a reduction of about $3 \%$ from ENDF/B-IV value.

\section{B. ENDF/B-V.1 (1983)}

${ }^{n a t} \mathbf{F e}[\mathbf{F u}(\mathbf{O R N L})]$ In the ENDF/B-V.2 release, the file was again revised:

- Inelastic scattering cross sections for 21 levels in ${ }^{57} \mathrm{Fe}$ and 2 levels in ${ }^{58} \mathrm{Fe}$ were included and lumped as 14 sections. Corresponding gamma-ray production data (file 12) and covariance data (file 13) were also provided.
- Arthur and Young evaluations [24] for $(\mathrm{n}, 2 \mathrm{n}),(\mathrm{n}, \mathrm{d})$, $(n, t)$, and $\left(n,{ }^{3} \mathrm{He}\right)$ were judged to be superior to ENDF/B-V and were adopted.

- Energy imbalances [25] were corrected from 2 to $20 \mathrm{MeV}$ by adjusting the total gamma-ray production cross sections (file 13) to reproduce estimated values of heating per collision. The adjusted results approach those of ENDF/B-IV. The largest changes occur in the energy range 13 to $20 \mathrm{MeV}$ where the adjusted results lie in between all available data [26-31].

- Resonance parameters (file2) and covariances (file 32 ) were revised so that the files and the corresponding document [32] are consistent. Changes are either very small or involve insignificant resonances.

\section{ENDF/B-V.2 (1985)}

- No changes

\section{ENDF /B-VI}

\section{A. ENDF/B-VI.0 (1990)}

\section{${ }^{54}$ Fe "Mod-1" [D.M. Hetrick, C.Y. Fu, N.M. Larson (ORNL)], aka "ENDF/B-V MOD-3"}

- This work employed nuclear model codes including the Distorted Wave Born Approximation (DWBA) program DWUCK and the Hauser-Feshbach code TNG [33-35]. The TNG code provides energy and angular distributions of particles emitted in the compound and pre-compound reactions, ensures consistency among all reactions, and maintains energy balance. Further details were referred to by the euphemism "Details pertinent to the contents of this evaluation will be published at a later date." (see $[36,37])$

- Resonance parameters and thermal cross sections are from Mughabghab [5] and adjusted through SAMMY by N. Larson using J.A. Harvey data [38] between 110 and $700 \mathrm{keV}$, capture cross section background in File 3 was based on Allen [11, 39]. Above $700 \mathrm{keV},{ }^{54} \mathrm{Fe}$ data from J.A. Harvey [38] used.

- Uncertainty files: $A n \mathrm{LB}=8$ section is included for all non-derived files as required by ENDF/B-VI.

${ }^{56}$ Fe "Mod-1" [D.M. Hetrick, C.Y. Fu, N.M. Larson (ORNL)], aka "ENDF/B-V MOD-3" ORNL-4617 [2] describes ENDF/B-III. Major revisions 
for ENDF/B-IV and -V are summarized in ORNL/TM7523 [40] and ORNL/TM-8283 [41]. The present evaluation is based on the calculations described in ORNL/TM9964 [37]. The major improvements over ENDF-5 MOD3 are:

1. Evaluation is now isotopic.

2. Analysis is based on a new TNG code much improved over the version used for $\mathrm{ENDF} / \mathrm{B}-\mathrm{V}$ for calculating energy-angle correlated spectra.

3. Resonance parameters are extended from $400 \mathrm{keV}$ to $850 \mathrm{keV}$ [42].

4. New data sets $[24,38,43-49]$ are incorporated.

5. Integral results [50-53] are considered for the (n,n') evaluation above $3 \mathrm{MeV}$.

6. Gamma-ray production cross sections and spectra are given for each reaction, including $\mathrm{MT}=51-75$, instead of for the nonelastic.

7. Recoil spectra are given in file-6.

8. Charged particle spectra are given.

${ }^{57}$ Fe "Mod-1" [D.M. Hetrick, C.Y. Fu, N.M. Larson (ORNL)], aka "ENDF/B-V MOD-3"

- This work employed the Hauser-Feshbach code TNG [33-35]. The TNG code provides energy and angular distributions of particles emitted in the compound and pre-compound reactions, ensures consistency among all reactions, and maintains energy balance. Details pertinent to the contents of this evaluation will be published at a later date.

- Resonance parameters and thermal capture cross section were taken from [5]. Some capture widths were adjusted to compromise with the averaged data of [39]. Low keV scattering cross sections were based on [54] which required an additional negative energy resonance. Two s-wave resonances above the resonance energy range were added to reduce the total cross sections between $150 \mathrm{keV}$ and 200 $\mathrm{keV}$. For total cross sections above $200 \mathrm{keV}$ and capture above $2 \mathrm{MeV}$, natural iron data of ENDF/B-V were used for the purpose of making sure that the total cross section minima in ${ }^{n a t} \mathrm{Fe}$ is maintained (when summing evaluations of isotopes together). For capture between $200 \mathrm{keV}$ and $2 \mathrm{MeV}$, linear interpolation between ref. [39] and ENDF/B-V were made.

- Uncertainty files: $\mathrm{An} \mathrm{LB}=8$ section is included for all non-derived files as required by ENDF/B-VI.

${ }^{58}$ Fe "Mod-1" [D.M. Hetrick, C.Y. Fu, N.M. Larson (ORNL)], aka "ENDF/B-V MOD-3"
- This work employed the Hauser-Feshbach code TNG [33-35]. The TNG code provides energy and angular distributions particles emitted in the compound and pre-compound reactions, ensures consistency among all reactions, and maintains energy balance. "Details pertinent to the contents of this evaluation will be published at a later date."

- Resonance parameters and thermal capture cross section were taken from [5], and adjusted to the data of Beer et al. [55] through the SAMMY code by N.M. Larson. Some capture widths were adjusted downward to compromise with the averaged data of [56]. Average capture data of [57] were used to generate capture background below $400 \mathrm{keV}$ and for the pointwise cross sections from $400 \mathrm{keV}$ to $2 \mathrm{MeV}$. Thermal capture and capture in the 10.4 $\mathrm{keV}$ resonance were further adjusted by SAMMY to agree with the ENDF/B-V dosimetry evaluation [58], which was adjusted to agree with integral data. Low keV scattering cross sections were based on $\mathrm{AP}=7.2 \mathrm{fm}$ given by [59]. For total cross sections above $400 \mathrm{keV}$ and capture cross sections above 2 $\mathrm{MeV}$, natural iron data of ENDF/B-V were used for the purpose of making sure that the total cross section minima in natural iron is maintained.

- Uncertainty files: $A n \mathrm{LB}=8$ section is included for all non-derived files as required by ENDF/B-VI.

\section{B. ENDF/B-VI.1 (1991)}

${ }^{54} \mathrm{Fe},{ }^{56} \mathrm{Fe}$ "Mod-2" [ORNL]

- Elastic transformation matrix removed

${ }^{57} \mathrm{Fe},{ }^{58} \mathrm{Fe}$ "Mod-2" [ORNL]

- The secondary particle distributions for $\mathrm{MF}=6$, $\mathrm{MT}=51-52$ were corrected-to-center of mass from laboratory coordinates. The elastic transformation matrix was removed.

C. ENDF/B-VI.2 (1993) -VI.3 (1995) -VI.4 (1996)

- No changes

\section{ENDF/B-VI.5 (1997-1998)}

\section{${ }^{54}$ Fe "Mod-3" [McLane (BNL)]}

- Update file 1, fix residual nucleus in $\mathrm{MF}=5$, $\mathrm{MT}=103$

${ }^{58}$ Fe "Mod-3" [McLane (BNL)]

- Corrected residual nucleus in File 6, MT=16. Corrected lower energy limit in File 6, MT=51,52 to agree with File 3 . Updated File 1 comments and corrected references. 


\section{E. ENDF/B-VI.6 (1998-1999)}

${ }^{54} \mathrm{Fe},{ }^{56} \mathrm{Fe},{ }^{57} \mathrm{Fe}$ "Mod-4" [Chadwick, Young, Koning (LANL/ECN)]

- LANL-150 extension to $150 \mathrm{MeV}$ (see [60-64])

- The covariance files $(\mathrm{MF}=33)$ were removed from the file.

\section{F. ENDF/B-VI.7 (1999-2000)}

${ }^{54} \mathrm{Fe},{ }^{56} \mathrm{Fe},{ }^{57} \mathrm{Fe}$ "Mod-5" [Frankle, Reedy, Young (LANL)]

- Update gamma-ray spectrum from (n,g) and adjust Q to ensure energy conservation [65]

${ }^{58}$ Fe "Mod-2" [Frankle, Reedy, Young (LANL)]

- Update gamma-ray spectrum from $(n, \gamma)$ and adjust Q to ensure energy conservation [65]

\section{G. ENDF/B-VI.8 (2001)}

- No changes

\section{ENDF/B-VII}

\section{A. ENDF/B-VII.0 (2006)}

${ }^{54} \mathrm{Fe},{ }^{57} \mathrm{Fe}$ [Trellue (LANL)]

- Minor correction to MF6 MT5

${ }^{56} \mathrm{Fe}$ [Trellue (LANL)]

- Remade MF6 MT5 using corrected GNASH code, to fix an earlier bug. Impact is reduced sec. particle prod. for new ENDF/B-VII release except for the $\alpha$ production cross section, whose factors were increased in Aug. 2006 to match unpublished Haight experimental data.

\section{B. ENDF/B-VII.1 (2011)}

${ }^{54} \mathbf{F e},{ }^{56} \mathbf{F e},{ }^{57} \mathbf{F e}$

- Kunieda (LANL/JAEA): alpha particle production cross sections and energy spectra added based on Iwamoto-Harada model.

- BNL: RR covariance added

- BNL: Covariance of thresholds adjusted

\section{ENDF/B-VIII}

\section{A. ENDF/B-VIII.0 $\beta 0$ (8 Apr. 2016)}

${ }^{56} \mathrm{Fe}$

- CIELO ${ }^{56} \mathrm{Fe}$ file from BNL, IAEA and CNDC

Add minor Fe isotopes from the CIELO collaboration $\left({ }^{54} \mathbf{F e},{ }^{57} \mathbf{F e}\right.$ and $\left.{ }^{58} \mathbf{F e}\right)$ New fast regions in all from EMPIRE calculations. New (currently undocumented) resonance regions in all:

- ${ }^{54} \mathrm{Fe}$, new Atlas values, in Reich-Moore format

- ${ }^{57} \mathrm{Fe}$, new Atlas values, in $\mathrm{LRF}=7$ format, ReichMoore approximation

- ${ }^{58} \mathrm{Fe}$, new evaluation from Moxon [7]

B. ENDF/B-VIII.0 $\beta 1$ (25 Apr. 2016)

${ }^{54} \mathbf{F e}$

- From CIELO Iron project (rev. 246 of CIELO Iron project)

${ }^{56} \mathrm{Fe}$

- Final before WPEC meetings (rev. 246 of CIELO Iron project)

${ }^{57} \mathrm{Fe}$

- From CIELO iron project (rev. 246 of CIELO Iron project)

C. ENDF/B-VIII.0 $\beta 2$ (19 Aug. 2016)

${ }^{54} \mathrm{Fe}$

- BNL: Revised ${ }^{54} \mathrm{Fe}$ from the CIELO project. This evaluation includes revised resonance region from IRSN up to $1.2 \mathrm{MeV}$ in the $\mathrm{LRF}=7$ format, EMPIRE calculation for fast region and capture cross section tuned to match average resonance cross section. Direct/Semi-Direct capture was added above the resonance region.

${ }^{56} \mathrm{Fe}$

- BNL/IRSN: Revised ${ }^{56} \mathrm{Fe}$ from the CIELO project. This evaluation includes revised resonance region from IRSN up to $850 \mathrm{keV}$ in the $\mathrm{LRF}=7$ format, elastic and inelastic cross sections and angular distributions from beta1 and EMPIRE calculation for the remainder of the fast region. 


\section{ENDF/B-VIII.0 $\beta 3$ (2 Nov. 2016)}

\section{${ }^{54} \mathrm{Fe}$ \\ - IRSN: resonances up to $1.036 \mathrm{MeV}$ \\ - BNL: EMPIRE calculation from $1.036 \mathrm{MeV}$ - 150 $\mathrm{MeV}$ \\ - BNL: (n,2n), (n,p) and (n,a) cross sections taken from IRDFF, partial reactions scaled to match these totals \\ ${ }^{56}$ Fe IAEA file fe56ib18b, IAEA-BNL Collabo- ration for CIELO}

- Resolved resonance range up to $850 \mathrm{keV}$ basically from JENDL-4.0

- The resonance energy at $766.7 \mathrm{keV}$ was corrected.

- The background near $800 \mathrm{keV}$ was reduced by 50 percent.

- Artificial "background" was added to capture around $24.5 \mathrm{keV}$, since capture in the hole of the elastic seems to be low and is HIGHLY SENSITIVE to many benchmarks. It was stipulated that the broad dip in this energy region is caused by inappropriately placed bound states. The adjusted capture cross section now follows the $1 / v$ behavior.

- The total cross section above the resonance range was taken from JEFF-3.2 because it is consistent with the Berthold [66] measurements on Fe-nat. The contribution of the minor isotopes is taken into account.

- The capture cross sections above $860 \mathrm{keV}$ were taken from the RPI data presented by Y. Danon at the ND2016 Conference.

- JEFF-3.2 contains inelastic cross sections measured by Dupont [67], but the authors discovered normalization problems and the results were never published. New measurements were performed by Negret [68], but with a lower resolution. The Dupont and Negret data were binned over a suitable energy mesh. A piecewise linear scaling parameter was constructed to adjust Dupont data such that they agree on average with the Negret data. It was also found that the energy calibration of Negret data did not match the resonances of the total cross section. A correction was made to the energy scale which amounted to $2.5 \mathrm{keV}$ at $1.8 \mathrm{MeV}$. Inelastic cross sections between $0.85 \mathrm{e} 6$ and $3.5 \mathrm{e} 6 \mathrm{eV}$ are taken from JEFF-3.2.

- The elastic cross section is defined as the difference between the total and the remaining partial cross sections. By resolution-broadening of the elastic cross section to 0.3 percent, good agreement is observed with the measured Kinney [69] data.
- The elastic angular distributions in the resolved resonance range were reconstructed from the resonance parameters. The Legendre moments were resolution-broadened with a resolution function of constant width of $1 \mathrm{keV}$. For most applications the resolution-broadened Legendre coefficients are sufficient, but the user still has the option to reconstruct detailed distributions from the resonance parameters. Above the resonance range up to $4 \mathrm{MeV}$ the angular distributions were taken from JEFF-3.2 because they accurately follow the Kinney data (with a correction for the minor isotopes).

- The P2 Legendre coefficients of elastic scattering were increased with a linear background, starting with 0 at $0.3 \mathrm{MeV}$, peaking to 0.7 at $0.85 \mathrm{MeV}$ and ending with 0 at $1.5 \mathrm{MeV}$.

- Similarly, the P4 Legendre coefficients of elastic scattering were increased linearly, starting with 0 at $0.5 \mathrm{MeV}$, peaking to 0.2 at $0.85 \mathrm{MeV}$ and ending with 0 at $1.5 \mathrm{MeV}$.

- At higher energies all cross sections, angular distributions and spectra are taken from EMPIRE calculation, which was tuned to reproduce the important reaction channels and radionuclide production data.

${ }^{57} \mathrm{Fe}$

- BNL: revised with new EMPIRE calculation for fast region

\section{E. ENDF/B-VIII.0 $\beta 3.1$ (19 Jan. 2017)}

${ }^{54} \mathrm{Fe}$

- Add in roughly 64 resonances from G. Giubrone's thesis [70] noted in Atlas evaluation but missing from IRSN evaluation

- Add high energy capture background to the RRR to account for missing p- \& d-wave resonances

${ }^{56} \mathbf{F e}$ New CIELO revision for Fe56, corresponding to IAEA version fe56ib19v. Major change: The elastic angular distributions in the resolved resonance range are taken from resolution-broadened Perey data [71]. Above the resonance range up to $2.5 \mathrm{MeV}$ the angular distributions correspond to re-fitted Kinney data [72] with some adjustments based on the comparison with Perey data in the overlapping region. In the range $2.5-4.0 \mathrm{MeV}$ the angular distributions are taken from Smith [73]. Above $2.5 \mathrm{MeV}$ the angular distributions are from the Empire calculation. 


\section{F. ENDF/B-VIII.0 $\beta 4$ (28 Feb. 2017)}

Fluctuations in Minor Iron Isotopes Red Cullen performed a COMPLOT comparison of beta3.1 and ENDF/B-VII.1 total cross sections for all minor Fe isotopes. It was clear from these figures that the fluctuations existing in ENDF/B-VII.1 were lost in the creation of the new CIELO evaluations.

- ${ }^{54} \mathrm{Fe}$, in ENDF/B-VII.1, the ${ }^{54} \mathrm{Fe}$ evaluation was tuned to match [38] using either GLUCS or SAMMY (not clear the actual method, but clearly was a least squares fit) above $700 \mathrm{keV}$.

- ${ }^{57} \mathrm{Fe}$ and ${ }^{58} \mathrm{Fe}$, in ENDF/B-VII.1, above $2 \mathrm{MeV}$ the ${ }^{57} \mathrm{Fe}(\mathrm{n}, \mathrm{tot})$ evaluation was a scaled natFe(n,tot) "to make sure the total cross section of natFe was maintained". Similarly, above $400 \mathrm{keV}$ the ${ }^{58} \mathrm{Fe}(\mathrm{n}$,tot) cross section was scaled for ENDF/B-VII.1.

These fluctuations have not been restored or replaced in the evaluations and they are unchanged from $\beta 3.1$.

${ }^{56} \mathbf{F e}$ This evaluation is very similar to the $\beta 3$ files. The file has been assembled at the IAEA and nicknamed 'fe56ib23w'. It consists of:

- EMPIRE calculation "ib23" (GForge repository No.529)

- Resolved resonance range up to $850 \mathrm{keV}$ basically from JENDL-4.0 (Froehner evaluation for JEF-2-2 with corrections for typos). The resonance energy at $766.7 \mathrm{keV}$ was corrected. The p-wave resonance at $59.5 \mathrm{keV}$ was removed. The background near 800 $\mathrm{keV}$ was reduced by 40 percent. Artificial "background" was added to capture around $24.5 \mathrm{keV}$, since capture in the hole of the elastic seems to be low and is HIGHLY SENSITIVE to many benchmarks. It was stipulated that the broad dip in this energy region is caused by inappropriately placed bound states. The adjusted capture cross section now nearly follows the $1 / \mathrm{v}$ behavior.

- The total cross sections above the resonance range were taken from JEFF-3.2, which is essentially the Vonach-Tagesen evaluation with superimposed fluctuations that correspond to Berthold measurements on Fe-nat. The contribution of the minor isotopes is taken into account.

- The capture cross sections above $860 \mathrm{keV}$ were taken from the RPI data presented by Y. Danon at the ND2016 Conference.
- JEFF-3.2 contains inelastic cross sections measured by Dupont et al., but the authors discovered normalization problems and the results were never published. New measurements were performed by Negret, but with a lower resolution. The Dupont and Negret data were binned over a suitable energy mesh. A piecewise linear scaling parameter was constructed to adjust Dupont data such that they agree on average with the Negret data. It was also found that the energy calibration of Negret data did not match the resonances of the total cross section. A correction was made to the energy scale which amounted to $2.5 \mathrm{keV}$ at $1.8 \mathrm{MeV}$.

In addition, the inelastic cross section below $1 \mathrm{MeV}$ was reduced by 15 percent to approximately agree with Perey data.

- The (n,p) partial cross sections are re-scaled to match the total $(\mathrm{n}, \mathrm{p})$ cross section in the IRDFFv1.05 file.

- The elastic cross section is defined as the difference between the total and the remaining partial cross sections. To test the consistency, the cross section was resolution-broadened to 0.3 percent, achieving good agreement with Kinney data.

- The elastic angular distributions in the resolved resonance range are taken from resolution-broadened Perey data. Above the resonance range up to 2.5 $\mathrm{MeV}$ the angular distributions correspond to refitted Kinney data with some adjustments that reduce the anisotropy, based on the comparison with Perey data in the overlapping region. In the energy range 2.5-4.0 MeV the angular distributions are taken from Smith (1973). Above $4 \mathrm{MeV}$ and below $35 \mathrm{keV}$ the angular distributions are from the Empire calculation.

\section{ACKNOWLEDGMENTS}

Work at Brookhaven National Laboratory was sponsored by the Office of Nuclear Physics, Office of Science of the U.S. Department of Energy under Contract No. DEAC02-98CH10886 with Brookhaven Science Associates, LLC.
[1] M. Herman, A. Trkov, R. Capote, G. P. A. Nobre, D. A. Brown, R. Arcilla, Y. Danon, A. Plompen, Q. Jing, G. Zhigang, L. Tingjin, L. Hanlin, R. Xichao, L. Leal,
T. Kawano, M. Sin, and K. Simakov, S.P.and Guber, "Evaluation of neutron reactions on iron isotopes for CIELO and ENDF/B-VIII.0," Nuclear Data Sheets 
(2018).

[2] S. K. Penny and W. E. Kinney, "A re-evaluation of natural iron neutron and gamma-ray production cross...," no. ORNL-4617 (1971). ENDF Report ENDF-139.

[3] C. Y. Fu and F. G. Perey, "Evaluation of neutron and gamma-ray-production cross sections for natural iron (ENDF/B-V MAT 1326)," Tech. Rep. ORNL/TM-7523, Oak Ridge National Laboratory (1980). ENDF Report ENDF-302.

[4] S. F. Mughabghab, Atlas of Neutron Resonances, Vol.1 and 2. Elsevier, Amsterdam (2017).

[5] S. F. Mughabghab, M. Divadeenam, and N. E. Holden, Neutron Cross Sections, Vol.1, Neutron Resonance Parameters and Thermal Cross Sections, Part A, $Z=1-60$. Academic Press, New York (1981).

[6] S. F. Mughabghab, Atlas of Neutron Resonances, 5ed., Resonance Parameters and Thermal Cross Sections, $Z=$ 1-100. Elsevier, Amsterdam (2006).

[7] M. Moxon Tech. Rep. INDC(UK)-089 rev. 1 (2005).

[8] C. M. Perey Private communication (to be published) (1977).

[9] F. Mann Private communication (1977).

[10] B. J. Allen, A. R. d. L. Musgrove, J. W. Boldeman, M. J. Kenny, and R. L. Macklin, "Resonance neutron capture in ${ }^{56}$ Fe," Nuclear Physics, Section A, 269, p. 408 (1976).

[11] B. J. Allen, A. R. d. L. Musgrove, J. W. Boldeman, and R. L. Macklin, "Valence neutron capture in ${ }^{54} \mathrm{Fe}, " \mathrm{Nu}-$ Clear Physics, Section A, 283, p. 37 (1977).

[12] C. Le Rigoleur, A. Arnaud, and J. Taste, "Mesures en valeur absolue des sections efficaces de capture radiativedes neutrons par le ${ }^{23} \mathrm{Na}, \mathrm{Cr},{ }^{55} \mathrm{Mn}, \mathrm{Fe}, \mathrm{Ni},{ }^{103} \mathrm{Rh}$, $\mathrm{Ta},{ }^{197} \mathrm{Au},{ }^{238} \mathrm{U}$ dans le domaine de $10 \mathrm{~A} 600 \mathrm{keV}, "$ Tech. Rep. 4788 (1976). Centre d'Etudes Nucleaires, Saclay Reports.

[13] W. E. Kinney and F. G. Perey, "High-resolution fastneutron gamma-ray production cross sections for iron up to $2100 \mathrm{keV}$," NuClear SCIENCE AND ENGINEERING, 63, p. 418 (1977).

[14] D. I. Garber and R. R. Kinsey, "Neutron cross sections. Volume II. Curves," Tech. Rep. BNL-325, Brookhaven National Laboratory (1976).

[15] W. E. Kinney and F. G. Perey Private communication (1977).

[16] G. T. Chapman and G. L. Morgan Tech. Rep. ORNLTM-5416, Oak Ridge National Laboratory (1976).

[17] N. D. Dudey and R. Kennerley, "Evaluation of Fe56(n,p)Mn-56 cross sections for ENDF/B-III," Tech. Rep. BNL-NCS-50446 (1975). In ENDF/B-IV Dosimetry File.

[18] D. L. Smith and J. W. Meadows, "Cross-section measurement of (n,p) reactions for ${ }^{27} \mathrm{Al},{ }^{46,47,48} \mathrm{Ti},{ }^{54,56} \mathrm{Fe},{ }^{58} \mathrm{Ni}$, ${ }^{59} \mathrm{Co}$, and ${ }^{64} \mathrm{Zn}$ from near threshold to $10 \mathrm{MeV}, " \mathrm{Nu}-$ Clear Science And EngineERing, 58, p. 314 (1975).

[19] H. Vonach, "Status of some activation cross sections for reactor neutron dosimetry in the range 13-15 MeV," Tech. Rep. IAEA-208 (1975).

[20] H. Vonach et al. in Conf. Nucl. Cross Sections And Tech., vol. 1 of NBS-SP-299, (Washington) (1968).

[21] N. C. Dyer and J. H. Hamilton, ${ }^{~}{ }^{56} \mathrm{Fe}$ and ${ }^{58} \mathrm{Fe}(\mathrm{n}, \mathrm{p})$ cross sections for 14.4 MeV neutrons," JournaL OF INOrganic And Nuclear Chemistry, 34, p. 1119 (1972).

[22] J. C. Robertson, B. N. Audric, and P. Kolkowski, "The ${ }^{56} \mathrm{Fe}(\mathrm{n}, \mathrm{p}){ }^{56} \mathrm{Mn}$ and ${ }^{27} \mathrm{Al}(\mathrm{n}, \alpha){ }^{24} \mathrm{Na}$ cross sections at 14.78 MeV," Journal of NuClear Energy, 27, p. 139
(1973).

[23] S. M. Qaim et al. Chemical Nucl. Data Conf., (Canterbury), p. 121 (1971).

[24] E. D. Arthur and P. G. Young, "Evaluation of neutron induced cross sections on ${ }^{54,56} \mathrm{Fe}$ to $40 \mathrm{MeV}$," Tech. Rep. LA-8626-MS, Los Alamos National Laboratory (1980). ENDF Report ENDF-304.

[25] R. E. MacFarlane, "Energy balance of ENDF/B-V," Trans. Am. Nucl. Soc., 33, p. 681 (1979).

[26] C. G. Hoot, V. J. Orphan, and J. John Tech. Rep. GULFRT-A-10743 (1971).

[27] V. J. Orphan, C. G. Hoot, and V. C. Rogers, "GammaRay Production Cross Sections for Iron from 0.86 to 16.7 MeV," Nuclear Science And Engineering, 57, p. 309 (1975).

[28] J. K. Dickens, G. L. Morgan, and F. G. Perey Tech. Rep. ORNL-TM-4798, Oak Ridge National Laboratory (1972).

[29] J. K. Dickens, G. L. Morgan, and F. G. Perey NucL. ScI. ENG., 50, p. 311 (1973).

[30] G. T. Chapman, G. L. Morgan, and F. G. Perey Tech. Rep. ORNL-TM-5416, Oak Ridge National Laboratory (1976).

[31] D. M. Drake, E. D. Arthur, and M. G. Silbert, "Cross Sections for Gamma-Ray Production by $14-\mathrm{MeV}$ Neutrons," Nuclear Science And Engineering, 65, p. 49 (1978).

[32] C. M. Perey and F. G. Perey, "Evaluation of resonance parameters for neutron interaction with iron isotopes for energies up to $400 \mathrm{keV}$," Tech. Rep. ORNL/TM-6405, Oak Ridge National Laboratory (1980). ENDF Report ENDF-298.

[33] C. Fu Nuclear Science And Engineering, 100 (1988).

[34] C. Fu, "Neutron cross sections from 10 to $50 \mathrm{MeV}$," (Upton, N.Y.) (May 1980). Brookhaven report BNL-NCS51425.

[35] K. Shibata and C. Fu Tech. Rep. ORNL/TM-10093, Oak Ridge National Laboratory (1986).

[36] D. M. Hetrick, C. Y. Fu, and N. M. Larson, "ENDF/BVI.1 evaluation of n + 54Fe," (1991).

[37] C.Y.Fu and D. Hetrick, "Update of the ENDF/B-V Mod3 iron: Neutron-producing reaction cross sections and energy-angle correlations," Tech. Rep. ORNL/TM-9964, Oak Ridge National Laboratory (1986). ENDF Report ENDF-341.

[38] J. Harvey. Personal communication (1989).

[39] B. J. Allen and A. R. d. L. Musgrove, "S-wave resonance parameters in the structural materials," in Neutron Data of Structural Materials for Fast Reactors (K. Böckhoff, ed.), (Oxford), pp. 447-475, OECD, Pergamon Press (1977). Proceedings of a Specialists' Meeting held at the Central Bureau for Nuclear Measurements, Geel, Belgium, 5-8 December, 1977.

[40] C. Y. Fu and F. G. Perey, "Evaluation of neutron and gamma-ray-production cross sections for natural iron (ENDF/B-V MAT 1326)," Tech. Rep. ORNL/TM-7523, Oak Ridge National Laboratory (1980). ENDF Report ENDF-302.

[41] C. Y. Fu, "Summary of the ENDF/B-V evaluations for carbon, calcium, iron, copper, and lead and ENDF/B-V Revision 2 for calcium and iron," Tech. Rep. ORNL/TM8283, Oak Ridge National Laboratory (1982). ENDF Report ENDF-325.

[42] C. M. Perey, F. G. Perey, J. A. Harvey, N. W. Hill, and N. M. Larson, " ${ }^{56} \mathrm{Fe}$ and ${ }^{60} \mathrm{Ni}$ resonance parameters," in Proc. Inter. Conf. Nuclear Data for Science and Technol- 
ogy (1992).

[43] A. Pavlik and H. Vonach, "Evaluation of the angle integrated cross sections from the interaction of $14 \mathrm{MeV}$ neutrons with medium and heavy nuclei," Tech. Rep. Physics Data 13-4 (1988).

[44] D. C. Larson in Nuclear Data for Basic and Applied Science: Proceedings of the International Conference, Santa Fe, New Mexico, 13-17 May 1985 (P. Young, ed.), no. v. 1 in Nuclear Data for Basic and Applied Science: Proceedings of the International Conference, Santa Fe, New Mexico, 13-17 May 1985, Gordon and Breach Science Publishers (1986).

[45] A. Marcinkowski, R. W. Finlay, G. Randers-Pehrson, C. E. Brient, R. Kurup, S. Mellema, A. Meigooni, and R. Tailor, "Neutron emission cross sections at 25.7 mev: ${ }^{51} \mathrm{~V},{ }^{56} \mathrm{Fe},{ }^{65} \mathrm{Cu},{ }^{93} \mathrm{Nb}$, and ${ }^{209} \mathrm{Bi}, "$ NuCleAr SCIENCE AND ENGINEERING, 83, p. 13 (1983).

[46] A. Takahashi, J. Yamamoto, T. Murakami, K. Oshima, H. Oda, K. Fujimoto, M. Ueda, M. Fukazawa, Y. Yanagi, J. Miyaguchi, and K. Sumita Tech. Rep. A-83-01, Osaka University (1983).

[47] C. Fu and D. Hetrick, "Experience in using the covariances of some ENDF/B-V dosimetry cross sections: proposed improvements and addition of cross-reaction covariances," in ASTM-EURATOM Symposium on Reactor Dosimetry, Gaithersburg, MD, USA, 22 Mar 1982, no. CONF-820321-15 (Jan. 1982).

[48] J. Frehaut et al. Tech. Rep. BNL-51245 (1980).

[49] S. M. Grimes, R. C. Haight, K. R. Alvar, H. H. Barschall, and R. R. Borchers, "Charged-particle emission in reactions of 15-mev neutrons with isotopes of chromium, iron, nickel, and copper," Physical Review, PART C, NuClEAR Physics, 19, p. 2127 (1979).

[50] R. E. Maerker, B. Broadhead, C. Y. Fu, et al. in Proceedings of the Conference on Nuclear Data for Basic and Applied Science, (Santa Fe), Gordon and Breach (1985).

[51] J. V. Pace Private communication (1985).

[52] S. N. Cramer and E. M. Oblow Tech. Rep. ORNL/TM5548, Oak Ridge National Laboratory (1976).

[53] R. H. Johnson et al. Trans. Am. Nucl. Soc., no. 22 (1975).

[54] A. Hibden Tech. Rep. ANL-4963, Argonne National Laboratory (1953). Data from V. McLane, Neutron Cross Sections, Vol. 2 (Academic Press, 1988).

[55] H. Beer, L. D. Hong, and F. Kappeler, "A measurement of the total neutron cross section of iron-58 in the energy range from 7 to $325 \mathrm{keV}$," NuCleAR SCIENCE AND ENGINEERING, 67, p. 184 (1978).

[56] B. J. Allen and R. L. Macklin, "Resonance neutron capture in ${ }^{58} \mathrm{Fe}, "$ Journal of Physics G: Nuclear Physics, 6, no. 3, p. 381 (1980).

[57] Y. N. Trofimov, "Radiative capture cross section of ${ }^{58} \mathrm{Fe}$ at a neutron energy of $0.5-2.0 \mathrm{MeV}$," ATOMnAYA ENERGIYA, 58, no. 4 (1985).

[58] R. E. Schenter, F. Schmittroth, and F. M. Mann, "Evaluations of the ${ }^{58} \mathrm{Fe}(n, \gamma){ }^{59} \mathrm{Fe}$ and ${ }^{54} \mathrm{Fe}(n, p){ }^{54} \mathrm{Mn}$ Reactions for the ENDF/B-V Dosimetry File," in Nuclear Cross Sections for Technology: Proceedings of the Inter- national Conference on Nuclear Cross Sections for Technology, Held at the University of Tennessee, Knoxville, TN, October 22-26, 1979, Volume 13 (J. L. Fowler, C. H. Johnson, and C. D. Bowman, eds.) (1980).

[59] J. B. Garg, S. Jain, and J. A. Harvey, "Neutron total cross section and resonance parameters of ${ }^{58} \mathrm{Fe}, "$ PHYs. REV. C, 18, pp. 1141-1147 (Sep 1978).

[60] M. B. Chadwick and P. G. Young Physical Review C, 47 (1993).

[61] E. D. Arthur and P. Young, "Evaluation of cross sections to $40 \mathrm{MeV}$ for ${ }^{54,56} \mathrm{Fe}$," in Proceedings of the Symposium on Neutron Cross Sections from 10 to $50 \mathrm{MeV}$ (M. R. Bhat and S. Pearlstein, eds.), BNL-NCS-51245, Brookhaven National Laboratory (May 1980).

[62] M. B. Chadwick and P. G. Young, "GNASH calculations of $\mathrm{n}, \mathrm{p}+54,56,57,58 \mathrm{Fe}$ and benchmarking of results," APT progress report: 1 August - 1 September 1996, Los Alamos National Laboratory (August 1996). Internal memo T-2-96/MS-52 from R.E. MacFarlane to L. Waters.

[63] M. B. Chadwick, P. G. Young, R. E. MacFarlane, and A. J. Koning, "High-energy nuclear data libraries for accelerator-driven technologies: Calculational method for heavy recoils," in Proceedings of the 2nd International Conference on Accelerator Driven Transmutation Technology and Applications, (Kalmar, Sweden) (June 1996).

[64] P. G. Young, E. D. Arthur, and M. Chadwick Tech. Rep. LA-12343-MS, Los Alamos National Laboratory (1992).

[65] S. C. Frankle, R. C. Reedy, and P. G. Young Tech. Rep. LA-13812, Los ALamos National Laboratory (2001).

[66] K. Berthold, C. Nazareth, G. Rohr, and H. Weigman, "Total cross section of natural Fe." Private communication (1994).

[67] E. Dupont, P. Ribon, H. Weigmann, and G. Vanpraet, "High resolution measurement of the neutron inelastic scattering crosssection of ${ }^{56} \mathrm{Fe}, "$ p. 529 (1997). Conf.on Nucl.Data for Sci.and Techn., Trieste 1997.

[68] A. Negret, C. Borcea, P. Dessagne, M. Kerveno, A. Olacel, A. J. M. Plompen, and M. Stanoiu, "Cross-section measurements for the ${ }^{56} \mathrm{Fe}(\mathrm{n}, \mathrm{xn} \gamma)$ reactions," PHYSICAL Review, Part C, Nuclear Physics, 90, p. 034602 (2014).

[69] W. E. Kinney and J. W. Mcconnell, "High resolution neutron scattering experiments at ORELA," p. 1319 (1976). Int.Conf.on Interact.of Neutr.with Nuclei,Lowell 1976.

[70] G. Giubrone, Neutron capture measurement of $54 \mathrm{Fe}$ and $57 F e$ at CERN n-TOF. PhD thesis, University of Valencia (2014).

[71] C. M. Perey, F. G. Perey, J. A. Harvey, N. W. Hill, and N. M. Larson, ${ }^{656} \mathrm{Fe}$ and ${ }^{60} \mathrm{Ni}$ resonance parameters," p. 41 (1991). Conf.on Nucl.Data for Sci.and Technol.,Juelich 1991.

[72] W. E. Kinney, Neutron Elastic and Inelastic Scattering from ${ }^{56} \mathrm{Fe}$ from 4.60 to $7.55 \mathrm{MeV}$. PhD thesis (1968). Thesis or dissertation.

[73] A. Smith and P. Guenther, "Scattering of mev neutrons from elemental iron," NuClear SCIENCE AND ENGINEERING, 73, p. 186 (1980). 